\title{
INFLUENCE DE DÉFOLIAISONS SUR LA STRUCTURE DU BOIS DE PIN MARITIME
}

\author{
H. POLGE et Simone GARROS \\ avec la collaboration technique de P. MICHEL \\ Station de Recherches sur la Qualité des Bois, \\ Central national de Recherches forestières, I.N.R.A., \\ 54 - Nancy
}

\section{RÉSUMÉ}

Dans un peuplement de Pins maritimes de 10 ans, 10 arbres ont été entièrement défoliés au début du printemps 1960 (référence C), 10 autres l'ont été également entièrement au début des printemps 1960 et 1961 (référence B), et 10 autres ont été à moitié défoliés au début des printemps 1960 et 1961 (référence A). L'incidence de ces traitements sur la structure du bois a été étudiée par comparaison avec 10 témoins (référence T).

L'analyse densitométrique a fait apparaître sur les arbres des traitements $\mathrm{B}$ et $\mathrm{C}$ une densité du bois de printemps exceptionnellement faible en 1960, et exceptionnellement élevée en 1961 ; la largeur des accroissements annuels a été fortement réduite à la suite des traitements; mais, au cours des années 1966 à 1969, celle des arbres du traitement A est devenue supérieure à celle des témoins, la différence étant significative en 1967. Le pourcentage de bois dense à l'intérieur des accroissements annuels augmente avec la sévérité des traitements.

Des études anatomiques montrent que la très faible densité du bois initial de 1960 est due uniquement à la minceur des membranes cellulaires, et non aux dimensions des lumens qui sont tout à fait normales; des considérations d'ordre physiologique permettent d'expliquer ce phénomène.

Des attaques virulentes d'insectes, même quand elles ne sont pas mortelles, peuvent causer aux arbres forestiers des troubles de croissance graves; cela se produit notamment dans les cas de pullulations de chenilles processionnaires (Thaumetopoea pityocampa) qui en arrivent à occasionner, sur différentes espèces du genre Pinus, la perte momentanée de tout l'appareil assimilateur.

Il est important, en une telle occurrence, de connaître l'incidence de ces dégâts sur l'équilibre biologique de l'arbre, et par voie de conséquence, sur la nature du bois élaboré.

L'étude dont il est rendu compte ici a été entreprise à l'instigation de R. JoLY dans le cadre d'une expérience de simulation sur Pins maritimes, assise par lui en forêt 
domaniale d'Hourtin, et dont les premiers résultats ont été publiés par ailleurs (R. JoLY, 1970).

Nous nous limiterons ici à une analyse détaillée de la structure des accroissements annuels produits, en rappelant cependant pour mémoire les effets spectaculaires des traitements sur la croissance (par exemple, perte d'un peu plus de 5 années $1 / 2$ de production sur 10 dans le cas d'un effeuillage complet).

\section{1. - MATÉrIEL ET MÉTHODE}

Le dispositif en cause comprenait: 10 arbres témoins (référence T), 10 arbres ayant subi un demi-effeuillage au début des printemps 1960 et 1961 (référence A), 10 arbres ayant subi un effeuillage total au début des printemps 1960 et 1961 (référence B), et 10 arbres ayant subi un effeuillage total au début du printemps 1960 seulement (référence C).

Ces divers traitements d'effeuillage artificiel étaient destinés à reproduire, suivant différentes modalités, les dégâts des chenilles processionnaires, tout en rendant plus facile qu'en cas de défoliaisons par les insectes, l'implantation d'un dispositif expérimental équilibré permettant une interprétation statistique des résultats.

Les effets des traitements sur la structure du bois ont été étudiés à l'aide d'une méthode doublement non destructive: l'analyse densitométrique, déjà décrite par ailleurs (PolgE, 1966).

Dans une première étape, deux carottes de sondage (références $\mathrm{E}$ et $\mathrm{S}$ ) ont été prélevées au printemps 1970 dans les 36 arbres encore vivants à l'époque; ces échantillons ont été ensuite radiographiés, puis leurs images explorées à l'aide dun microdensitomètre, en utili sant un rapport d'agrandissement de $20 \mathrm{X}$ et une fente d'exploration de $0,3 \mathrm{~mm}$ dans le sens tangentiel et 10 microns dans le sens radial; dans des conditions bien définies d'exposition aux rayons $X$ et d'analyse, les graphiques ainsi obtenus peuvent être gradués directement en densité du bois.

En fait, le traitement le plus sévère (traitement B) a entraîné la production de cernes extrêmement fins, et même durant plusieurs années, consécutives ou non, l'absence totale d'accroissement; les courbes densitométriques ont permis dans de nombreux cas d'identifier ces cernes anornaux, mais il restait dans certains autres une marge d'incertitude qui interdisait d'analyser de façon rigoureuse les effets de cette modalité.

Les comparaisons statistiques, dont il sera fait état plus loin, n'ont donc porté que sur les traitements $T$, $A$ et $C$, et pour chaque individu, sur celui des deux profils $E$ ou $S$ qui correspondait à la meilleure des deux images radiographiques obtenues.

Ont été étudiées sur ces enregistrements les sept caractéristiques suivantes: densité maximale annuelle, densité minimale annuelle, largeur de cerne, largeur et pourcentage de bois de densité supérieure à 600 et à $700 \mathrm{~g} / \mathrm{dm}^{\prime 3}$, et ce pour les douze années écoulées, de 1958 à 1969 inclusivement. De cette façon, on disposait des renseignements concernant deux années : 1958 et 1959 antérieures aux défoliaisons, ce qui permettait de tester l'homogénéité des témoins et des arbres traités avant le début de l'expérience.

La radiographie et les profils densitométriques ayant fait apparaitre des effets véritablement spectaculaires sur les arbres correspondant aux modalités $\mathbf{B}$ et $\mathbf{C}$, des études de caractère anatomique ont été entreprises sur coupes microscopiques afin de pouvoir préciser le phénomène :

Des mesures de cavités cellulaires et d'épaisseurs de membranes ont ainsi été effectuées sur 4 arbres traités et 4 témoins ; elles ont intéressé les cernes 1959 et 1960, et pour chacun d'eux ont été déterminées les dimensions moyennes des cinq premières cellules du bois de printemps, le long de 25 alignements radiaux successifs, ainsi que les dimensions moyennes des membranes séparant la première de la deuxième et la deuxième de la troisième fibre, également pour 25 alignements radiaux successifs. Des tests de comparaison de moyennes ont permis de voir sur quelle caractéristique les traitements avaient exercé un effet significatif. 


\section{2. - RÉSULTATS}

\section{1. - Examen radiographique}

Sur la figure 1 sont regroupés, à titre d'exemple, les positifs au grossissement $4 \mathrm{X}$, de quatre échantillons caractéristiques: 1 témoin, 1 échantillon ayant subi le traitement $\mathrm{A}$, et 2 échantillons ayant subi le traitement $\mathrm{C}$; on voit que le traitement modéré par demi-effeuillage a entraîné une diminution notable de largeur des accroissements, mais il n'apparaît à l'œil nu aucune différence dans le noircissement des clichés correspondant à des différences de densité du bois; sur les deux arbres ayant subi un effeuillage complet, l'infiuence sur la croissance se manifeste avec une plus grande acuité, mais surtout se révèle un effet exceptionnellement marqué sur le bois de printemps de l'année 1960, beaucoup plus clair sur le positif que celui de tous les autres accroissements annuels des quatre échantillons; ceci correspond à une image radiographique très foncée, et donc à un bois de très faible densité.

L'incidence du traitement B est naturellement encore plus nette, mais l'extrême étroitesse de certains cernes n'aurait rendu possible la reproduction photographique des radiographies qu'en utilisant des agrandissements difficilement compatibles avec le format de la publication.
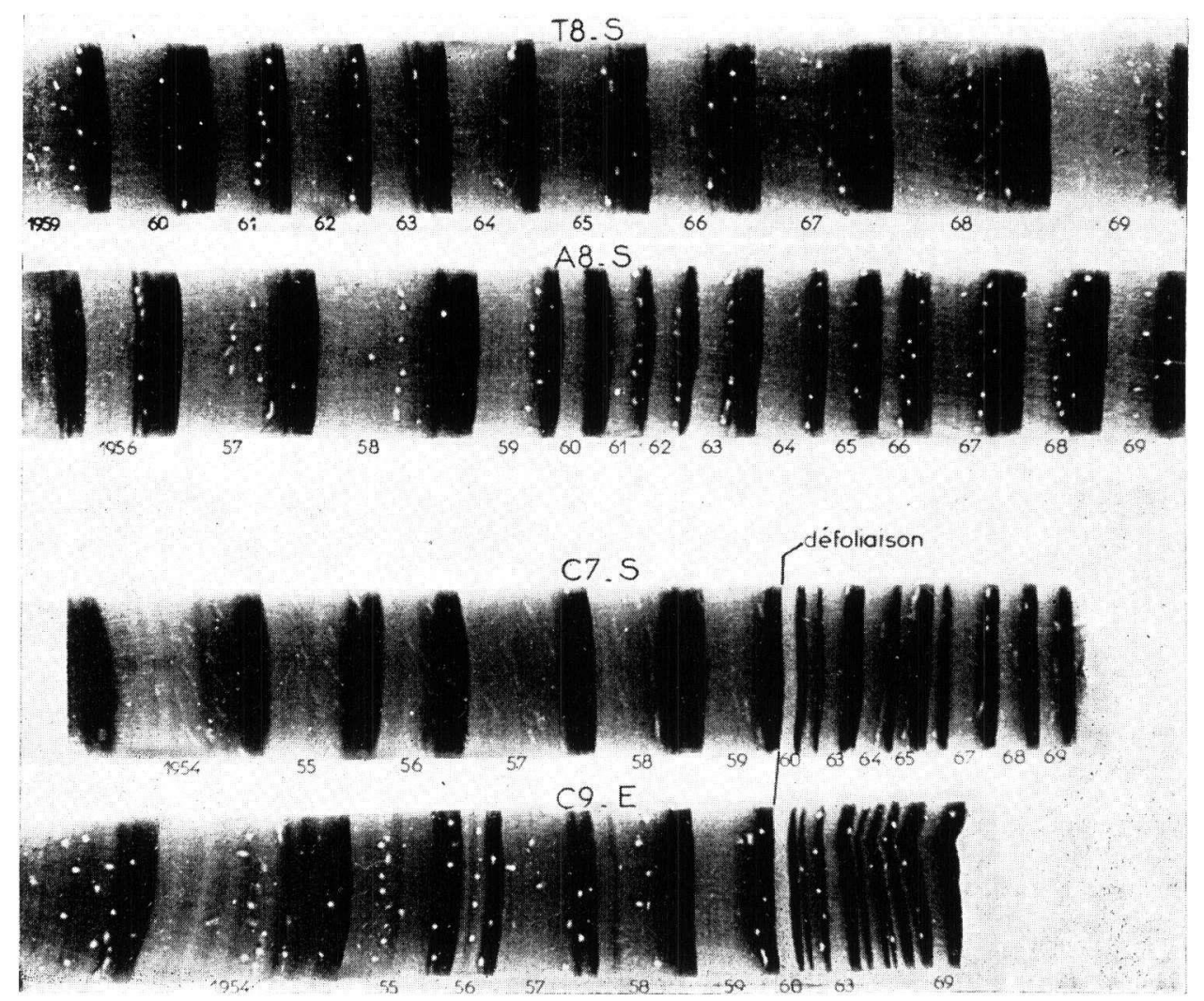

FIg 1. - Positifs de radiographie au grossissement 4 d'un témoin, d'un arbre du traitement $A$ et de deux arbres du traitement $C$

FIG. 1. - X-ray photograph positive prints of one control tree, one tree $A$ and two trees $C$ (enlargement $4 X$ 


\section{2. - Profils densitométriques}

Sur la figure 2 sont réunis les profils densitométriques de 5 échantillons caractéristiques pour les témoins et le traitement $\mathrm{A}$, de 2 échantillons du traitement $\mathrm{B}$ et de 8 du traitement $C$.

On peut y voir la confirmation sous forme quantitative des observations faites sur les radiographies elles-mêmes en ce qui concerne les réductions de croissance, plus ou moins sévères suivant les modalités, et surtout la densité du bois initial de 1960 qui, pour les traitements $\mathrm{B}$ et $\mathrm{C}$, est représentée par un pic d'une ampleur tout à fait exceptionnelle vers le bas des graphiques, c'est-à-dire vers les faibles densités.

\section{3. - Etude statistique}

Elle a porté sur l'ensemble des caractéristiques densitométriques relevées sur 10 arbres témoins, 10 arbres du traitement $\mathrm{A}$ et 8 du traitement $\mathrm{C}$.

Sur la figure 3 sont regroupées, sous forme graphique, les valeurs moyennes annuelles des principaux critères étudiés pour chaque modalité (il n'est pas fait état des résultats concernant les largeurs de bois de densité supérieure à 600 et à $700 \mathrm{~g} / \mathrm{dm}$ : qui, dans une certaine mesure, font double emploi avec les largeurs totales et les pourcentages correspondants). Un signe conventionnel indique les différences qui sont significatives au seuil de $5 \%$ d'une part et de $1 \%$ d'autre part.

On peut vérifier tout d'abord que tous les arbres étudiés étaient homogènes en 1958 et 1959, aussi bien pour la largeur des accroissements que pour les critères se rapportant à la densité du bois.

Quant aux effets des traitements, on peut faire les observations suivantes:

Densité maximale annuelle.

Il n'existe aucune différence significative entre témoins et arbres effeuillés, mais on doit cependant noter, pour les trois premières années ayant suivi le traitement, une baisse très nette des densités maximales pour le traitement $\mathrm{C}$ par rapport aux deux autres modalités.

Durant toute la période étudiée, témoins et arbres du traitement A ont eu des densités maximales annuelles vraiment très voisines.

Densité minimale annuelle.

Les seules différences significatives concernent les années 1960 et 1961 pour lesquelles la densité minimale du traitement $\mathrm{C}$ est très inférieure dans le premier cas, et très supérieure dans le deuxième cas, à la fois à celle des témoins et des arbres du traitement A. Les valeurs moyennes exactes sont, dans l'ordre T A C : 342, 351 et $267 \mathrm{~g} / \mathrm{dm}^{*}$ en $1960,356,358$ et $434 \mathrm{~g} / \mathrm{dm}^{3}$ en 1961 .

Par la suite, la densité minimale annuelle du traitement C demeure supérieure, et souvent assez nettement, à celle des deux autres modalités, mais les différences ne sont pas significatives, vraisemblablement en raison d'une insuffisance de l'échantillonnage. 


\section{Expérience de défoliaison}

DENSITES MAXIMALES ANNUELLES

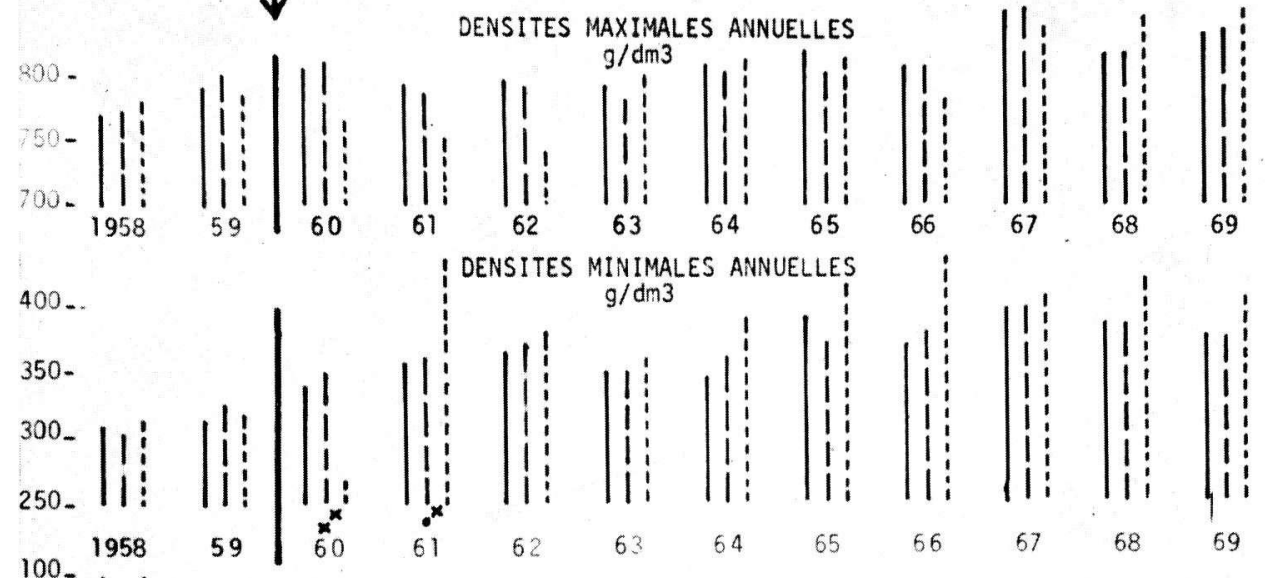

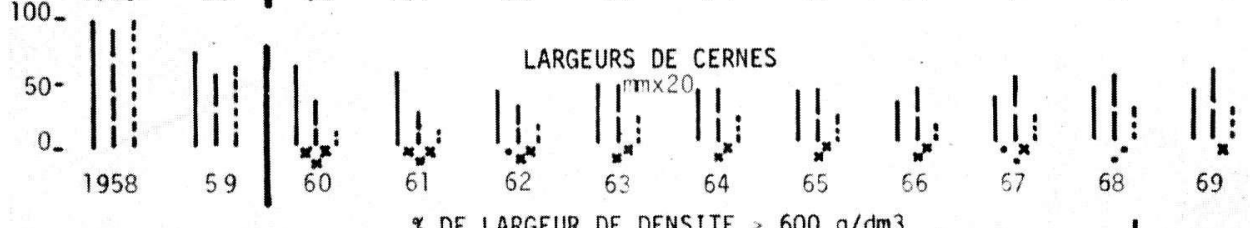

\% De LARGeUR DE DENSITE > $600 \mathrm{~g} / \mathrm{dm} 3$
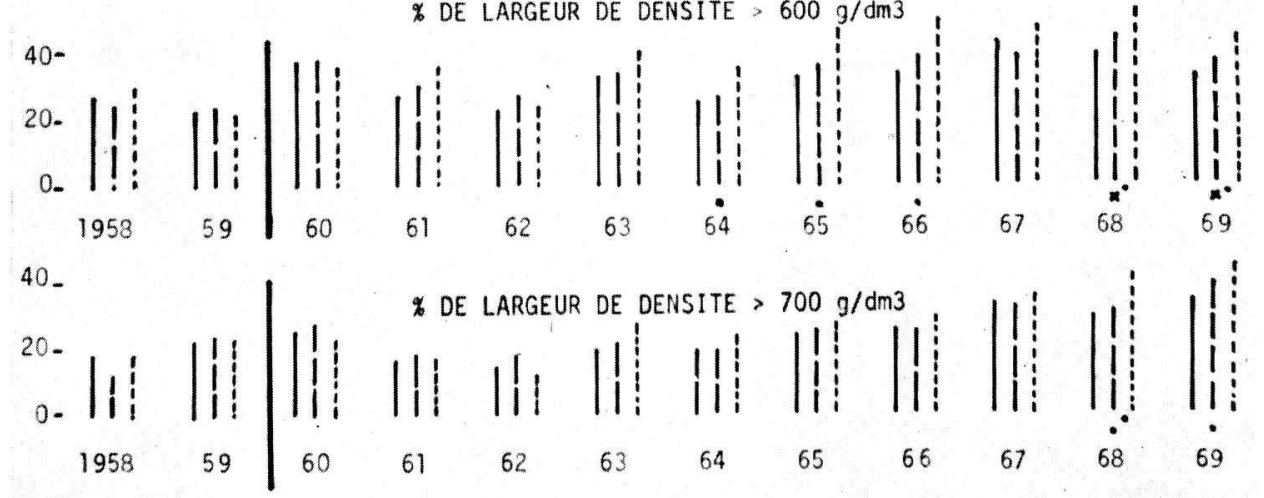

Têmoins

I Traitement $A$

12 demi-effeuillages

Traitement C

Effeuillage complet

\section{DIFFERENCES SIGNIFICATIVES}

au seuil de $5 \%$

au seuil de $1 \%$

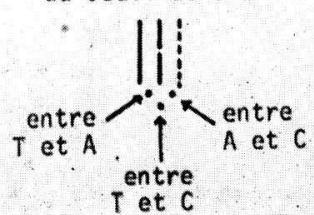

FIG. 3. - Comparaison entre témoins et arbres des traitements $A$ et $C$ au point de vue caractéristiques densitométriques et largeur de cernes

FIG. 3. - Comparison $A$ and $C$ treatments with control trees for ringwidths and 4 density criteria Expérience de défoliaison: Experience of defoliation; Densité maximale annuelle: Maximum annual densities; Densité minimale annuelle: Minimum annual densities; Largeur de cernes: Ringwidths; $\%$ de largeur de densité $>$ : Percentage of ringwidth having a density higher than; Témoins: Control trees; Traitement: Treatment; Demi-effeuillage: Halt defoliations; Effeuillage complet: Entire defoliations; Différences significatives au seuil de: Significant differences at the level; Entre $T$ et $A$ : 
Largeur de cernes.

C'est, tout naturellement, la caractéristique pour laquelle l'effet du traitement est le plus net; c'est la seule qui fait apparaître des différences significatives entre le traitement le plus modéré (modalité A) et les témoins, et ceci pour les trois années consécutives 1960, 1961 et 1962 ; pour les quatre années suivantes, la largeur des cernes du traitement $\mathbf{C}$ demeure significativement inférieure, à la fois à celle du traitement $\mathbf{A}$ et des témoins.

Par la suite, la croissance de $\mathrm{C}$ reste toujours inférieure à celle de $\mathrm{A}$ (tantôt au seuil de $5 \%$, tantôt au seuil de $1 \%$ ), mais en 1969 , elle ne diffère pas de façon significative de celle des témoins; cependant le fait le plus remarquable est la supériorité de la croissance des arbres du traitement A par rapport aux témoins, qui commence à se manifester en 1966, et qui est même significative en 1967, année où les arbres du traitement $A$ ont poussé de $2,6 \mathrm{~mm}$ sur le rayon contre $1,9 \mathrm{~mm}$ pour les témoins.

Ces résultats, bien qu'étonnants, concordent avec ceux publiés par R. JoLY (1970, op. cit.) sous forme de graphique portant sur la circonférence totale à hauteur d'homme aux divers âges; bien que la précision obtenue soit moins bonne, notamment du fait que les mesures ont dû être faites sur écorce, on observe que la pente de la courbe de croissance au cours des dernières années est plus forte pour la modalité D. 2 (qui correspond ici à la référence A) que pour les témoins.

\section{Pourcentage de bois dense à l'intérieur des accroissements annuels.}

Sauf exception, la hiérarchie des trois modalités est la même pour les pourcentages de bois de densité supérieure à $600 \mathrm{~g} / \mathrm{dm}$ : d'une part, et $700 \mathrm{~g} / \mathrm{dm}^{3}$ d'autre part.

D’une façon générale, les défoliaisons ont amené une augmentation de ces pourcentages, mais celle-ci ne s'est manifestée qu'assez tard.

$\mathrm{Au}$ niveau $600 \mathrm{~g} / \mathrm{dm}^{3}$, le traitement $\mathrm{C}$ donne un pourcentage significativement supérieur aux témoins à partir de 1964 et jusqu'à la fin, à la seule exception de l'année 1967, mais la discrimination s'accentue en 1968 et 1969 où C est également significativement différent de A.

Les différences sont moins nettes au niveau de $700 \mathrm{~g} / \mathrm{dm}^{3}$, les seuls écarts significatifs, de même sens que les précédents, ne concernent que les années 1968 et 1969.

\section{4. - Caractéristiques anatomiques}

Observations relatives au bois de printemps des années 1960 et 1961.

Les courbes densitométriques font apparaître une très faible densité pour le bois initial de 1960, mais également une densité anormalement élevée pour celui de 1961.

Bien que réduite, la largeur du cerne 1960 est cependant suffisante pour que le spot du microdensitomètre ait à coup sûr exploré sans risque d'erreur le point de plus faible densité ; par contre, le bois initial de 1961 est, sur la plupart des arbres des traitements B et C, extrêmement fin, et la question pouvait se poser de savoir si la forte densité minimale de ce cerne n'était pas due au nombre extrêmement réduit de 
cellules de bois de printemps qui aurait pu faire que la fente d'exploration chevauche simultanément, d'abord le bois final de 1960 et le bois initial de 1961, puis le bois initial de 1961 et le bois final de la même année; un certain nombre de coupes anatomiques ont donc été faites pour vérifier qu'il n'en était pas ainsi.

Sur la figure 4 sont regroupées 4 microphotographies (1 pour le traitement B, 3 pour le traitement $C$ ) dont la qualité n'est pas très satisfaisante, car les coupes microscopiques en cause ont dû être obtenues à partir de carottes de sondage de $5 \mathrm{~mm}$ de diamètre exceptionnellement fragiles.

Elles permettent cependant de faire des observations intéressantes:

Tout d'abord, pour les deux échantillons B $5 \mathrm{~S}$ et C $4 \mathrm{E}$, les traitements ont entraîné une désorganisation marquée du plan ligneux du bois de printemps de 1960, avec des perturbations très nettes dans les alignements radiaux des trachéides.

En outre, on observe dans le bois initial de 1960 une extrême minceur des membranes cellulaires, qui explique une densité minimale très faible; de même, les fibres du bois de printemps de 1961 (et, dans une moindre mesure, des années suivantes) apparaissent comme anormalement épaisses, ce qui prouve que sa densité élevée, trouvée au microdensitomètre, correspond bien à la réalité, et n'est pas due, comme on pouvait le craindre, à des défauts d'exploration.

Comparaison des caractéristiques biométriques concernant le bois de printemps des années 1959 et 1960.

De toutes les observations faites sur les arbres traités, celles relatives au bois initial de 1960 sont, à coup sûr, les plus inattendues, et il a paru intéressant de voir en quoi ses cellules constitutives se différenciaient de celles de l'année précédente, c'est-àdire du bois produit avant traitement; des calculs statistiques n'auraient pas été nécessaires pour vérifier que l'épaisseur de ses membranes était significativement différente de celle des autres années, mais il n'en était pas de même pour les dimensions des cellules, car une illusion d'optique bien connue veut que des éléments à parois minces paraissent avoir une taille supérieure à celle d'éléments à parois épaisses de mêmes dimensions.

Les résultats des mesures faites sur 200 épaisseurs de membranes d'arbres traités d'une part, et de témoins d'autre part, et sur les dimensions radiales de 500 cellules d'arbres témoins et 375 d'arbres traités sont consignés dans le tableau ci-dessous :

Caractéristiques

\begin{tabular}{|c|c|c|c|c|c|}
\hline \multirow{2}{*}{$\begin{array}{l}\text { Double épaisseur moyenne } \\
\text { des membranes }(e n \mu \mathrm{m}) \ldots .\end{array}$} & 1959 & $\left\{\begin{array}{l}\text { Témoins } \\
\text { Traités }\end{array}\right.$ & $\ldots \ldots \ldots \ldots$ & $\begin{array}{l}9,9 \\
8,5\end{array}$ & $-5,29$ \\
\hline & 1960 & $\left\{\begin{array}{l}\text { Témoins } \\
\text { Traités }\end{array}\right.$ & $\ldots \ldots \ldots \ldots$ & $\begin{array}{l}9,4 \\
6,1\end{array}$ & $-14,43$ \\
\hline \multirow{2}{*}{$\begin{array}{l}\text { Dimensions radiales moyen- } \\
\text { nes des fibres }(\text { en } \mu \mathrm{m}) \ldots \ldots\end{array}$} & 1959 & $\left\{\begin{array}{l}\text { Témoins } \\
\text { Traités }\end{array}\right.$ & $\ldots \ldots \ldots \ldots \ldots$ & $\begin{array}{l}44,6 \\
45,0\end{array}$ & 0,53 \\
\hline & 1960 & $\left\{\begin{array}{l}\text { Témoins } \\
\text { Traités }\end{array}\right.$ & $\ldots \ldots \ldots \cdots$ & $\begin{array}{l}44,1 \\
42,5\end{array}$ & $-1,85$ \\
\hline
\end{tabular}
de moyennes

Test « $t »$ de comparaison 

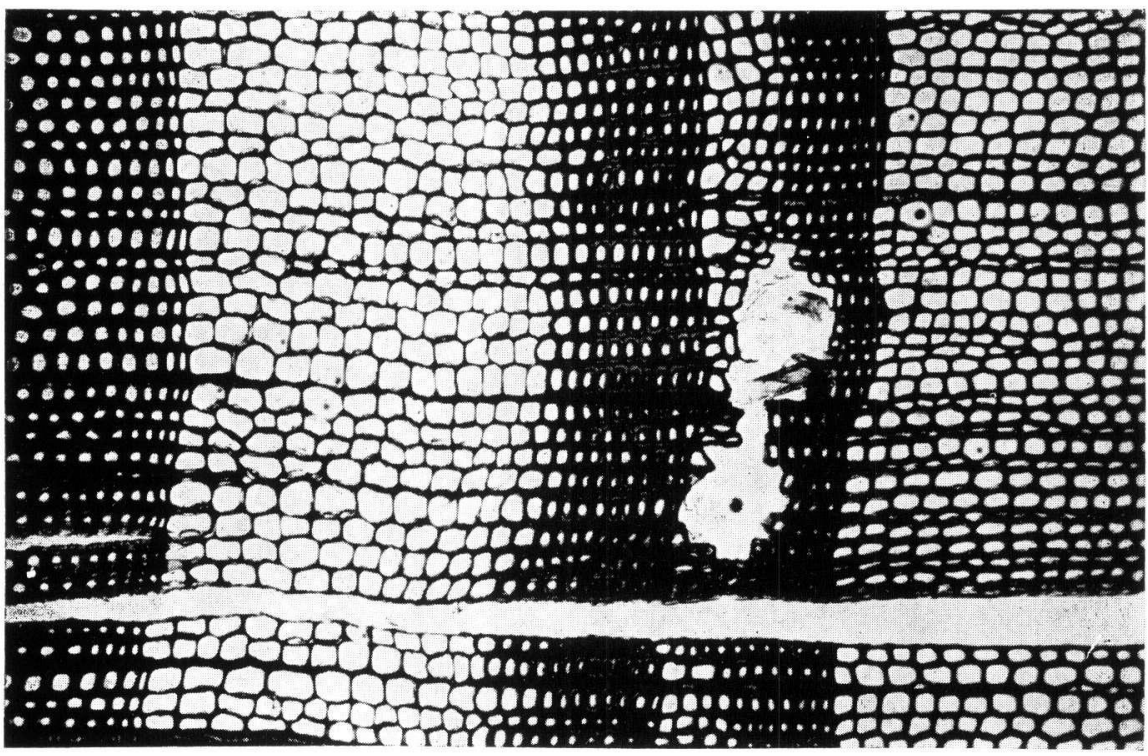

Echantillon CIS

1960

1961

1962

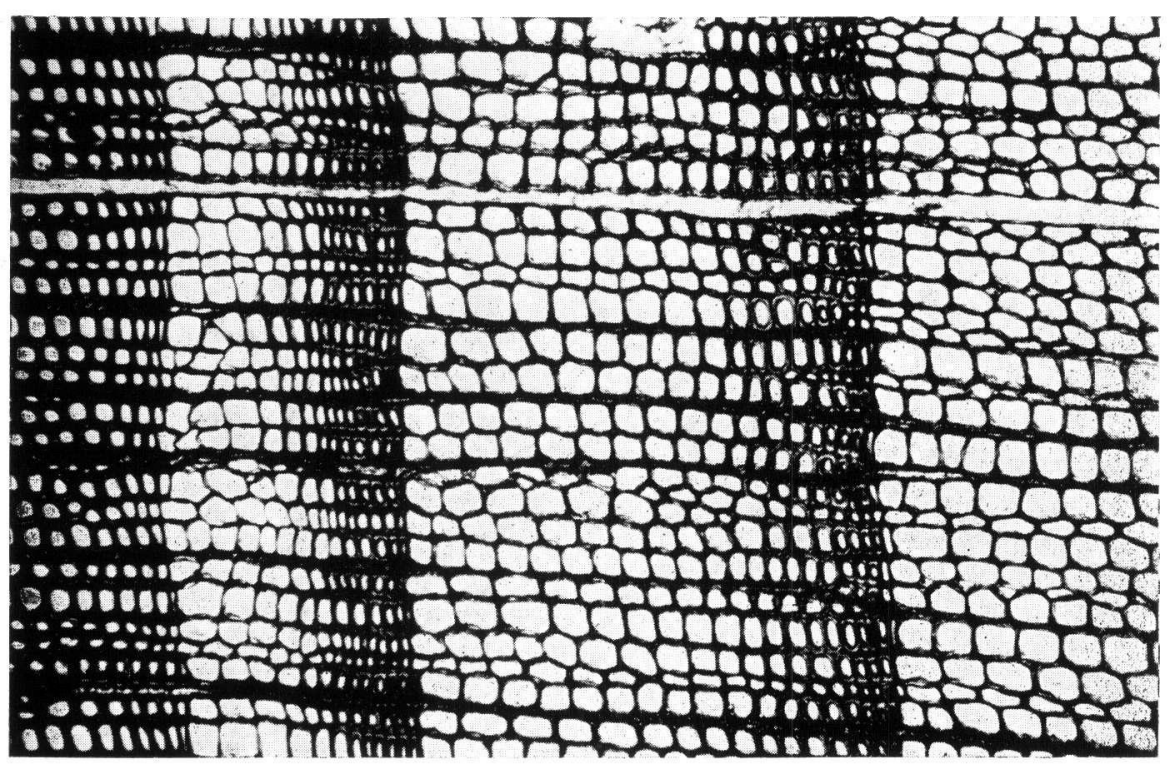

Echantillon C 4 E

FIG. 4. - Microphotographies (grossissement $85 \times$ )

FIG. 4. - Microphotographs enlarged 85 times

Echantillon: Sample 


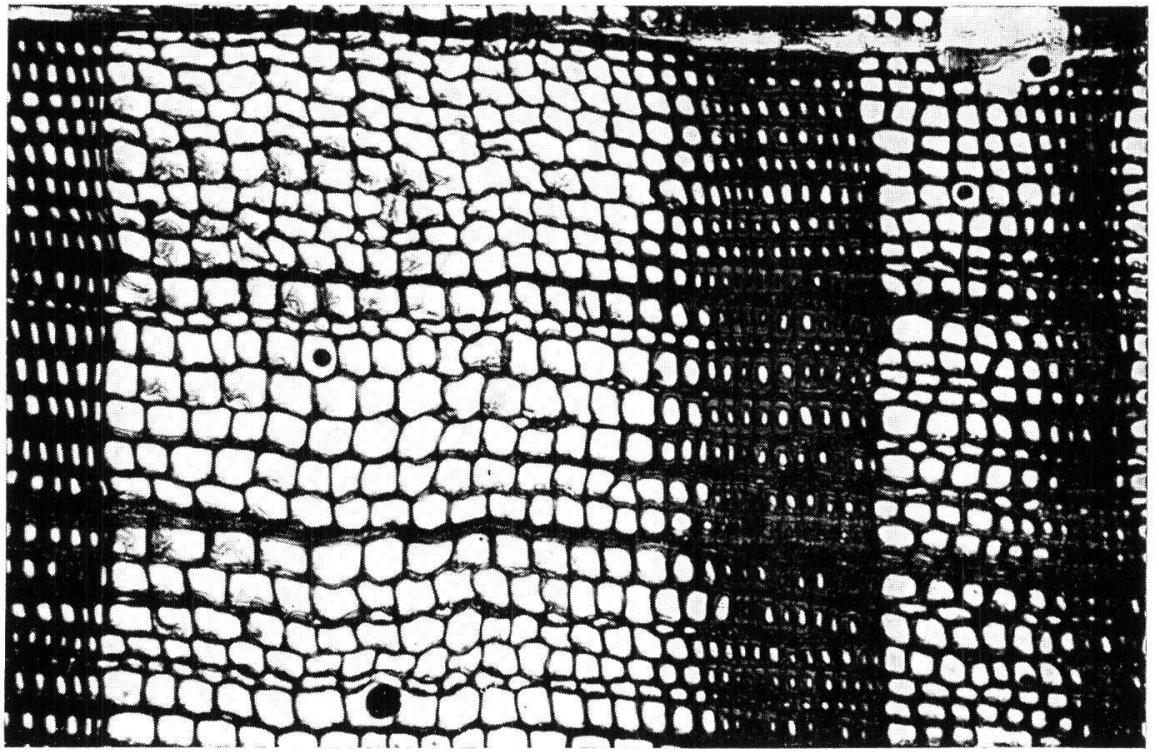

Echantillon $C 7$ E

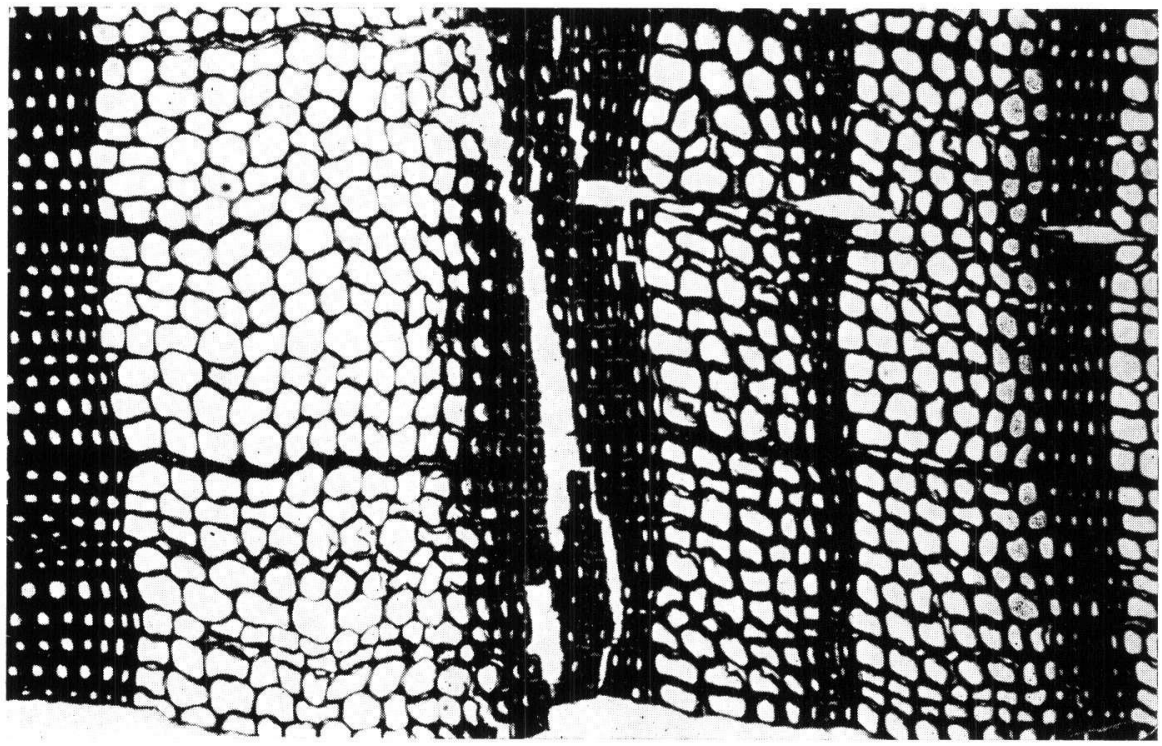


Il n’y a pas de différence significative entre arbres traités et témoins en ce qui concerne les dimensions radiales des fibres du bois de printemps, que ce soit avant ou après les défoliaisons.

En revanche, l'effet du traitement sur l'épaisseur des membranes de ce même bois de printemps est manifeste, puisque la valeur de cette caractéristique est, en 1960, pour les arbres traités, inférieure de $35 \%$ à celle des témoins, alors qu'avant le début de l'expérience, cette différence ne représentait, en valeur relative, que $14 \%$.

La faible densité du bois initial de 1960 pour les arbres totalement effeuillés n'est donc pas due à la plus grande taille des cellules, mais seulement à la plus faible épaisseur des membranes.

\section{3. - CONCLUSIONS}

Plusieurs enseignements d'ordre physiologique sont à tirer de cette expérience.

Tout d'abord, malgré un effeuillage complet, les arbres du traitement $\mathrm{C}$, et même ceux du traitement $\mathbf{B}$, ont fait en 1960 des accroissements qui sont loin d'être négligeables; certes, les bourgeons n'avaient pas été supprimés et ont donné naissance, dès le début de la saison de végétation 1960, à de nouvelles aiguilles qui ont pu rapidement permettre une reprise de l'activité photosynthétique.

Mais il convient d'observer, qu'aussi bien pour la modalité A que pour la modalité C, la largeur de l'accroissement 1961 a été plus faible que celui de 1960 : $1,27 \mathrm{~mm}$ contre $1,82 \mathrm{~mm}$ et $0,42 \mathrm{~mm}$ contre $0,59 \mathrm{~mm}$ respectivement.

Il semble bien qu'il faille voir là un effet de i'influence des réserves accumulées dans les cellules de parenchyme au début de l'expérience, qui se sont trouvées épuisées par la croissance de 1960 , et dont l'importance dépasserait celle des hydrates de carbone élaborés par l'ensemble des aiguilles produites durant cette même année.

Quant à la croissance supérieure constatée durant les dernières années dans les arbres du traitement A par rapport aux témoins (et dont, en ce qui concerne tout au moins l'année 1967, il n'y a pas 5 chances sur 100 pour qu'elle soit due au hasard), elle pourrait être attribuée au rajeunissement forcé de la masse foliaire qui aurait entraîné une augmentation du rendement de la photosynthèse.

La très faible densité minimale du cerne 1960 dans les arbres ayant subi les traitements $\mathrm{B}$ et $\mathrm{C}$ est sans doute plus facile à expliquer: on sait maintenant, à la suite notamment des expériences de LARson (1960), que la formation de cellules à grands lumens et à membranes minces du bois initial est directement liée à l'élaboration d'auxines dans les bourgeons en voie d'élongation, puisque d'une part la destruction de ces bourgeons entraîne de façon prématurée la production de cellules de bois final, et qu'inversement une application d'acide indol-acétique sur des plants ainsi décapités s'accompagne d'une formation limitée de cellules de plus grande taille à membranes plus minces ; quant au mode d'action de l'auxine, elle interviendrait, d'après les théories les plus récentes (Ordin et al., 1956), comme plastifiant de la membrane cellulaire, c'est-à-dire que, lorsqu'elle parvient en quantité accrue à l'assise cambiale, et lorsque l'approvisionnement en eau n'exerce pas lui-même un effet limitant, une même masse 
de substances constitutives des membranes cellulaires permettrait de produire des fibres de plus grande taille, donc à parois plus minces.

Dans le cas présent, il semble que l'apport d'auxines aurait été en 1960 à peu prês normal, puisque les bourgeons n'avaient pas été touchés, alors que l'approvisionnement en hydrates de carbone était fortement limité, ne pouvant, au début de la période de végétation tout au moins, se faire qu'aux dépens des réserves; c'est ce double phénomène qui rendrait compte des caractéristiques tout à fait anormales des trachéides du bois initial de 1960.

En ce qui concerne les années ultérieures, il n'est pas illogique de penser que l'activité des méristèmes apicaux, donc l'élaboration d'hormones de croissance, s'est trouvée considérablement ralentie du fait de l'épuisement des arbres en cause à la suite des traitements très sévères subis en 1960 ; la situation aurait ainsi été totalement renversée par rapport à l'année précédente, d'où la production de fibres plus petites à parois plus épaisses, donc d'un bois initial beaucoup plus dense.

\section{Reçu pour publication en février 1971.}

\section{SUMMARY}

EFFECTS OF ARTIFICIAL DEFOLIATIONS UPON THE WOOD STRUCTURE OF MARITIME PINE

Within a 10 years old maritime Pine stand,

- 10 trees (ref. C) were entirely defoliated during the 1960 early spring,

- 10 trees (ref. B) were entirely defoliated during the 1960 and 1961 early spring,

- 10 trees (ref. A) were half defoliated during the 1960 and 1961 early spring,

and their growth were compared with 10 control trees (ref. T). 1970).

The principal results have been reported by the author of this experience (R. JoLY,

The wood structure has been studied by X-raying $5 \mathrm{~mm}$ increment cores (fig. 1); the $X$ ray photographies have been scanned by a double beam microdensitometer, using a 10 microns slit; on the densitometric records (fig. 2) have been measured from 1958 to 1969 (i.e. from 2 years before treatments until 10 years after) the maximum and minimum annual densities, the ringwidths, and the percentages of ringwidths having a density higher than 600 and $700 \mathrm{~g} / \mathrm{dm}^{3}$; the results of the comparisons between control and treated trees are summarized in fig. 3 .

There is no significant difference for maximum annual densities.

The minimum annual densities of the trees $C$ are in 1960 very significantly lower and in 1961 very significantly higher than that of trees $\mathrm{T}$ and $\mathrm{A}$.

The ringwidths of $\mathrm{C}$ and A significantly differ for all the period 1960-1969, but in 1969, there is no significant difference between $\mathrm{C}$ and $\mathrm{T}$. The control trees have wider ring than the trees A from 1960 to 1962; T and A have roughly the same ringwidth from 1963 to 1965, and after 1965, the growth of control trees have been slower than that of the trees A, the difference being significant in 1967.

The percentages of dense wood within rings increases from $\mathrm{T}$ to $\mathrm{A}$ and to $\mathrm{C}$ for the period 1963 to 1969 , more significantly during the last years.

The ring 1960 has a width which is noticeable for the $\mathrm{C}$ and $\mathrm{B}$ treatments; it this shows the importance of foods stored in the parenchyma cells.

The earlywood density for B and C is exceptionally low in 1960 ; this is due (fig. 4 and comparison of mean values on table page 201) to the very thin cellwalls and not to the lumen diameters. It appears that the amount of auxin was not too much reduced, since the buds were not cut in these experiments; thus, after the physiological considerations of OrDIN (1958), the auxin induced plasticization of the wall was sufficent, but the synthesis of cell wall constituents was low, and so the lumina are normal, but the walls of earlywood are thin. 


\section{ZUSAMMENFASSUNG}

DER EINFLUSS DER ENTNADELUNG AUF DIE HOLZSTRUKTUR DER SEESTRANDKIEFER

In einem zehnjährigen Bestand der Seestrandkiefer (Pinus pinaster Ait.) wird der Einfluss der Entnadelung auf die Holzstruktur an je zehn Bäumen in den folgenden vier Behandlungen untersucht:

- T : unbehandelt (Nullfäche).

- A : zur Hälfte entnadelt im Frühjahr 1960 und 1961.

— B : vollständig entnadelt im Frühjahr 1960 und 1961.

- C : vollständig entnadelt im Frühjahr 1960.

Die densitometrische Analyse zeigt, dass die Bäume der Behandlungen B und C im Jahre 1960 eine aussergewöhnlich geringe, und im Jahre 1961 eine aussergewöhnlich hohe Frühholzdichte aufweisen. Die Behandlungen A, B und C hatten in den ersten Jahren nach der Entnadelung sehr stark reduzierte Jahrringbreiten. In den Jahren 1966 bis 1969 ergab sich jedoch bei der Behandlung A eine grössere Jahrringbreite als bei den unbehandelten Vergleichsstämmen; der Unterschied war im Jahre 1967 signifikant. Der Anteil an dichterem Holz innerhalb eines Jahrringes nimmt mit steigender Entnadelung zu.

Die anatomischen Untersuchungen zeigen, dass die sehr geringe Frühholzdichte im Jahre 1960 ausschliesslich auf die geringe Zellwandstärke und nicht auf die völling normalen Lumenabmessungen zurückzuführen ist; dies kann mit physiologischen Überlegungen erklärt werden.

\section{RÉFÉRENCES BIBLIOGRAPHIQUES}

Joly R., 1970. Action des déprédations dues aux insectes défoliateurs sur le Pin maritime (Pinus pinaster). Revue Forestière Française, $\mathrm{n}^{\circ}$ spécial La lutte biologique en forêt.

LARSON Ph. R., 1960. A physiological consideration of the springwood summerwood transition in Red Pine. Forest Science, 6, (2).

Ordin L., Applewhite T. H., Bonner J., 1956. Auxin-induced water uptake by avena coleoptile sections. Plant. Physiol., 31, 44-53.

Polge H., 1966. Etablissement des courbes de variation de la densité du bois par exploration densitométrique de radiographies d'échantillons prélevés à la tarière sur des arbres vivants. Applications dans les domaines technologiques et physiologiques. Thèse Docteur ès-Sciences. Faculté des Sciences de Nancy. Annales des Sciences Forestières, 23' (1). 